\title{
Intercalibration of the barrel electromagnetic calorimeter of the CMS experiment at start-up
}

The CMS Electromagnetic Calorimeter Group

P. Adzic,${ }^{v}$ R. Alemany-Fernandez, ${ }^{q}$ N. Almeida, ${ }^{q}$ G. Anagnostou, ${ }^{i, 1}$ D. Andelin, ${ }^{a g}$ M. Anfreville, ${ }^{f}$ I. Anicin, ${ }^{v, 2}$ Z. Antunovic, ${ }^{c}$ R. Arcidiacono, ${ }^{o}$ M. W. Arenton, ${ }^{a g}$

E. Auffray, ${ }^{w}$ S. Argiro,${ }^{o}$ A. Askew, ${ }^{a n}$ O. Atramentov, ${ }^{a n}$ S. Baccaro, ${ }^{n, 3}$ S. Baffioni, ${ }^{g}$ M. Balazs, ${ }^{a g}$ D. Barney, ${ }^{w}$ L. M. Barone, ${ }^{n}$ A. Bartoloni, ${ }^{n}$ C. Baty, ${ }^{h}$ D. Bandurin, ${ }^{a i}$ S. Beauceron, ${ }^{w}$ K.W. Bell, ${ }^{a c}$ R. Benetta, ${ }^{w}$ M. Bercher, ${ }^{g}$ C. Bernet, ${ }^{g}$ U. Berthon, ${ }^{g}$ M. Besancon, ${ }^{f}$ B. Betev, ${ }^{y}$ R. Beuselinck, ${ }^{a d}$ C. Biino, ${ }^{o}$ J. Blaha, ${ }^{h}$ P. Bloch, ${ }^{w}$ S. Blyth,,$z$ A. Bornheim, ${ }^{a l}$ J. Bourotte, ${ }^{g}$ A.M. Brett, ${ }^{y}$ R.M. Brown, ${ }^{a c}$ D. Britton, ${ }^{a d}$ M. Buehler, ${ }^{a g}$ P. Busson, ${ }^{g}$ B. Camanzi, ${ }^{a c}$ T. Camporesi, ${ }^{w}$ E. Carrera, ${ }^{a n}$ N. Cartiglia, ${ }^{o}$ F. Cavallari, ${ }^{n}$ M. Cerutti, ${ }^{g}$ P. Chang, ${ }^{a}{ }^{a}$ Y.H. Chang, ${ }^{z}$ C. Charlot, ${ }^{g}$ E.A. Chen, ${ }^{z}$ W.T. Chen, ${ }^{z}$ Z. Chen, ${ }^{y}$ R. Chipaux, ${ }^{f}$ B.C. Choudhary, ${ }^{k}$ R.K. Choudhury, ${ }^{l}$ D.J.A. Cockerill, ${ }^{a c}$ C. Combaret, ${ }^{h}$ S. Conetti,,$^{a g}$ F. Cossutti, ${ }^{p}$ B. Cox, ${ }^{a g}$ D.G. Cussans, ${ }^{a b}$ I. Dafinei, ${ }^{n}$ D.R. Da Silva Di Calafiori, ${ }^{y}$ G. Daskalakis, ${ }^{i}$ G. Davatz, ${ }^{y}$ A. David, ${ }^{q}$ K. Deiters, ${ }^{x}$ M. Dejardin, ${ }^{f}$ M. Djordjevic, ${ }^{v}$ R. Della Negra, ${ }^{h}$ G. Della Ricca, ${ }^{p}$ D. Del Re,${ }^{n}$

A. De Min, ${ }^{m}$ D. Denegri, ${ }^{f}$ P. Depasse,${ }^{h}$ J. Descamps,,${ }^{f}$ M. Diemoz, ${ }^{n}$ E. Di Marco, ${ }^{n}$ G. Dissertori, ${ }^{y}$ M. Dittmar, ${ }^{y}$ L. Djambazov, ${ }^{y}$ L. Dobrzynski, ${ }^{g}$ S. Drndarevic, ${ }^{v, 2}$ J.E. Duboscq, ${ }^{a h}$ D. Dutta, ${ }^{l}$ M. Dzelalija, ${ }^{c}$ A. Elliott-Peisert, ${ }^{w}$ H. El Mamouni, ${ }^{,}$ I. Evangelou, ${ }^{j}$ D.L. Evans, ${ }^{a b}$ B. Fabbro, ${ }^{f}$ J.L. Faure,${ }^{f}$ J. Fay, ${ }^{h}$ F. Ferri, ${ }^{f}$ P.S. Flower ${ }^{a c}$ D. Franci, ${ }^{n}$ G. Franzoni, ${ }^{a j}$ K. Freudenreich, ${ }^{y}$ W. Funk, ${ }^{w}$ S. Ganjour, ${ }^{f}$ C. Gargiulo, ${ }^{n}$ S. Gascon, ${ }^{h}$ M. Gataullin, ${ }^{a l}$ Y. Geerebaert, ${ }^{g}$ F.X. Gentit, ${ }^{f}$ Y. Gershtein, ${ }^{a n}$ A. Ghezzi, ${ }^{m, w}$ M.D. Ghodgaonkar, ${ }^{l}$ J. Gilly, ${ }^{g}$ A. Givernaud, ${ }^{f}$ S. Gninenko, ${ }^{s}$ A. Go, ${ }^{z}$ B. Gobbo, ${ }^{p}$ N. Godinovic, ${ }^{b}$ N. Golubev, ${ }^{s}$ D. Gong, ${ }^{a j, 4}$ P. Govoni, ${ }^{m}$ N. Grant, ${ }^{a b}$ P. Gras, ${ }^{f}$ R.J.S. Greenhalgh, ${ }^{a c}$ L. Guevara Riveros, ${ }^{g}$ J.P. Guillaud, ${ }^{e}$ M. Haguenauer, ${ }^{g}$ G. Hamel de Monchenault, ${ }^{f}$ M. Hansen, ${ }^{w}$ H.F. Heath, ${ }^{a b}$ B. Heltsley, ${ }^{a h}$ J.A. Hill, ${ }^{a c}$ W. Hintz, ${ }^{y}$ R. Hirosky, ${ }^{a g}$ P.R. Hobson, ${ }^{a e}$ A. Honma, ${ }^{w}$ G.W.S. Hou, ${ }^{a a}$ Y. Hsiung, ${ }^{a}{ }^{a}$ M. Husejko, ${ }^{q}$ B. Ille, ${ }^{h}$ R. Imlay, ${ }^{a g}$ Q. Ingram,${ }^{x}$ P. Jarry, ${ }^{f}$ C. Jessop,${ }^{a k}$ D. Jovanovic,${ }^{v, 2}$ K. Kaadze, ${ }^{a i}$ V. Kachanov, ${ }^{u}$ S. Kailas, ${ }^{l}$ S.K. Kataria, ${ }^{l}$ B.W. Kennedy, ${ }^{a c}$ K. Kloukinas, ${ }^{w}$ P. Kokkas, ${ }^{j}$ T. Kolberg, ${ }^{a k}$ N. Krasnikov, ${ }^{s}$ D. Krpic, ${ }^{v, 2}$ Y. Kubota, ${ }^{a j}$ P. Kumar, ${ }^{l}$ C.M. Kuo, ${ }^{z}$ P. Kyberd, ${ }^{a}$ A. Kyriakis,${ }^{i}$ M. Lebeau, ${ }^{w, 5}$ P. Lecomte, ${ }^{y}$ P. Lecoq, ${ }^{w}$ A. Ledovskoy, ${ }^{a g}$ G. Leshev, ${ }^{y}$ M. Lethuillier, ${ }^{h}$ S.W. Lin, ${ }^{a a}$ W. Lin, ${ }^{z}$ A.L. Lintern, ${ }^{a c}$ V. Litvine, ${ }^{a l}$ E. Locci, ${ }^{f}$ A.B. Lodge,${ }^{a c}$ E. Longo, ${ }^{n}$ D. Loukas, ${ }^{i}$ P.D. Luckey, ${ }^{y}$ W. Lustermann, ${ }^{y}$ C. Lynch, ${ }^{a b}$ 
Y. Ma, ${ }^{a l}$ H. Mahlke-Krueger, ${ }^{a h}$ M. Malberti, ${ }^{m}$ J. Malclès, ${ }^{f}$ D. Maletic, ${ }^{\nu}$ I. Mandjavidze, ${ }^{f}$ N. Manthos, ${ }^{j}$ Y. Maravin, ${ }^{a i}$ C. Marchica,,${ }^{x y}$ N. Marinelli, ${ }^{a k}$ A. Markou, ${ }^{i}$ C. Markou, ${ }^{i}$ M. Marone,${ }^{o}$ H. Mathez, ${ }^{h}$ V. Matveev,${ }^{s}$ C. Mavrommatis, ${ }^{i}$ G. Maurelli, ${ }^{h}$ P. Meridiani, ${ }^{w}$ P. Milenovic, ${ }^{v, y}$ G. Milleret,${ }^{g}$ P. Miné, ${ }^{g}$ A.K. Mohanty, ${ }^{l}$ F. Moortgat, ${ }^{y}$ M. Mur, ${ }^{f}$ P. Musella, ${ }^{q}$ Y. Musienko, ${ }^{s, a f}$ A. Nardulli, ${ }^{y}$ J. Nash, ${ }^{a d}{ }^{a}$ P. Nedelec, ${ }^{e}$ P. Negri, ${ }^{m}$ H.B. Newman, ${ }^{a l}$ A. Nikitenko, ${ }^{a d, 6}$ F. Nessi-Tedaldi, ${ }^{y}$ M.M. Obertino, ${ }^{o, 7}$ G. Organtini, ${ }^{n}$ T. Orimoto, ${ }^{a l}$ M. Paganoni, ${ }^{m}$ P. Paganini, ${ }^{g}$ A. Palma, ${ }^{n}$ B. Panev,${ }^{y, 9}$ L. Pant, ${ }^{l}$ A. Papadakis, ${ }^{d}$ I. Papadakis, ${ }^{i}$ I. Papadopoulos,${ }^{j}$ R. Paramatti, ${ }^{n}$ P. Parracho, ${ }^{q}$ N. Pastrone, ${ }^{o}$ J.R. Patterson, ${ }^{a h}$ F. Pauss, ${ }^{y}$ E. Petrakou, ${ }^{i}$ D.G. Phillips II, ${ }^{a g}$ P. Piroué, ${ }^{a m}$ F. Ptochos, ${ }^{d}$ I. Puljak, ${ }^{b}$ A. Pullia, ${ }^{m}$ T. Punz, ${ }^{y}$ J. Puzovic, ${ }^{v, 2}$ S. Ragazzi, ${ }^{m}$ S. Rahatlou, ${ }^{n}$ J. Rander, ${ }^{f}$ P.A. Razis, ${ }^{d}$ N. Redaelli, ${ }^{m}$ D. Renker, ${ }^{x}$ S. Reucroft, ${ }^{a f}$ J.M. Reymond,${ }^{f}$ P. Ribeiro, ${ }^{q}$ U. Roeser, ${ }^{y}$ C. Rogan, ${ }^{a l}$ T. Romanteau, ${ }^{g}$ F. Rondeaux, ${ }^{f}$ M. Ronquest,${ }^{a g}$ A. Rosowsky, ${ }^{f}$ C. Rovelli, ${ }^{n}$ P. Rumerio, ${ }^{w, 8}$ R. Rusack, ${ }^{a j}$ S.V. Rusakov, ${ }^{t}$ M.J. Ryan, ${ }^{a d}$ L. Sala, ${ }^{m}$ R. Salerno, ${ }^{m}$ F. Santanastasio, ${ }^{n, 8}$ D. Schinzel,,${ }^{y}$ C. Seez, ${ }^{a d}$ P. Sharp, ${ }^{w, a d}$ C.H. Shepherd-Themistocleous, ${ }^{a c}$ C. Siamitros, ${ }^{a e}$ D. Sillou, ${ }^{e}$ A. Singovsky, ${ }^{a j}$ Y. Sirois, ${ }^{g}$ A. Sirunyan,${ }^{a}$ J. Silva, ${ }^{q}$ P. Silva, ${ }^{q}$ J.G. Shiu, ${ }^{a}$ R.K. Shivpuri, ${ }^{k}$ P. Shukla,${ }^{l}$ B.J. Smith, ${ }^{a c}$ V.J. Smith, ${ }^{a b}$ M. Sproston, ${ }^{a c}$ F. Stöckli, ${ }^{y}$ H. Suter,${ }^{y}$ J. Swain, ${ }^{a f}$

T. Tabarelli de Fatis, ${ }^{m, *}$ M. Takahashi, ${ }^{a d}$ V. Tancini, ${ }^{m}$ A. Tcheremoukhine, ${ }^{r}$ O. Teller, ${ }^{w}$

K. Theofilatos, ${ }^{i}$ C. Thiebaux,${ }^{g}$ V. Timciuc,,${ }^{a l}$ C. Timlin, ${ }^{a d}$ M. Titov, ${ }^{f}$ A. Tobias, ${ }^{a g}$

A. Topkar, ${ }^{l}$ F.A. Triantis, ${ }^{j}$ S. Troshin, ${ }^{l}$ N. Tyurin, ${ }^{u}$ K. Ueno, ${ }^{a a}$ A. Uzunian, ${ }^{u}$

J. Varela,,$^{q, w}$ P. Verrecchia,${ }^{f}$ J. Veverka, ${ }^{a l}$ P. Vichoudis, ${ }^{w}$ T. Virdee, ${ }^{w, a d}$ E. Vlassov, ${ }^{w, 6}$ H.P. Von Gunten, ${ }^{y}$ M. Wang, ${ }^{a a}$ D. Wardrope, ${ }^{a d}$ M. Weber, ${ }^{y}$ J. Weng, ${ }^{y}$

J.H. Williams, ${ }^{a c, \dagger}$ Y. Yang, ${ }^{a l}$ I. Yaselli, ${ }^{a e}$ R. Yohay, ${ }^{a g}$ A. Zabi, ${ }^{g}$ N. Zamiatin, ${ }^{r}$

S. Zelepoukine, ${ }^{u, y}$ J. Zhang, ${ }^{a j}$ L.Y. Zhang, ${ }^{a l}$ K. Zhu ${ }^{a l}$ and R.Y. Zhu ${ }^{a l}$

${ }^{a}$ Yerevan Physics Institute, Yerevan, Armenia

${ }^{b}$ Technical University of Split, Split, Croatia

${ }^{c}$ University of Split, Split, Croatia

${ }^{d}$ University of Cyprus, Nicosia, Cyprus

${ }^{e}$ Laboratoire d'Annecy-le-Vieux de Physique des Particules,

IN2P3-CNRS, Annecy-le-Vieux, France

${ }^{f}$ DSM/DAPNIA, CEA/Saclay, Gif-sur-Yvette, France

${ }^{g}$ Laboratoire Leprince-Ringuet, Ecole Polytechnique, IN2P3-CNRS, Palaiseau, France

${ }^{h}$ Institut de Physique Nucléaire de Lyon, Université Lyon 1, CNRS/IN2P3, Villeurbanne, France

iInstitute of Nuclear Physics "Demokritos”, Aghia Paraskevi, Greece

${ }^{j}$ University of Ioánnina, Ioánnina, Greece

${ }^{k}$ University of Delhi, Delhi, India

${ }^{l}$ Bhabha Atomic Research Centre, Mumbai, India

${ }^{m}$ Istituto Nazionale di Fisica Nucleare e Università degli Studi di Milano Bicocca, Milano, Italy

${ }^{n}$ Sapienza Università di Roma e Sezione dell'INFN, Roma, Italy

${ }^{o}$ Università di Torino e Sezione dell'INFN, Torino, Italy

${ }^{p}$ Università di Trieste e Sezione dell'INFN, Trieste, Italy

${ }^{q}$ Laboratório de Instrumentação e Física Experimental de Partículas, Lisboa, Portugal

rJINR, Dubna, Russia

${ }^{s}$ Institute for Nuclear Research, Moscow, Russia

${ }^{t}$ Lebedev Physical Institute, Moscow, Russia 
"IHEP, Protvino, Russia

${ }^{v}$ Vinca Institute of Nuclear Sciences, Belgrade, Serbia

${ }^{w}$ CERN, European Organisation for Nuclear Research, Geneva, Switzerland

${ }^{x}$ Paul Scherrer Institut, Villigen, Switzerland

${ }^{y}$ Institute for Particle Physics, ETH Zurich, Zurich, Switzerland

${ }^{z}$ National Central University, Chung-Li, Taiwan

${ }^{a a}$ National Taiwan University (NTU), Taipei, Taiwan

${ }^{a b}$ University of Bristol, Bristol, United Kingdom

${ }^{a c}$ Rutherford Appleton Laboratory, Didcot, United Kingdom

ad Imperial College, University of London, London, United Kingdom

${ }^{a e}$ Brunel University, Uxbridge, United Kingdom

${ }^{a f}$ Northeastern University, Boston, Massachusetts, U.S.A.

${ }^{a g}$ University of Virginia, Charlottesville, Virginia, U.S.A.

${ }^{a h}$ Cornell University, Ithaca, New York, U.S.A.

${ }^{a i}$ Kansas State University, Manhattan, Kansas, U.S.A.

${ }^{a j}$ University of Minnesota, Minneapolis, MN, U.S.A.

${ }^{a k}$ University of Notre Dame, Notre Dame, IN, U.S.A.

${ }^{a l}$ California Institute of Technology, Pasadena, California, U.S.A.

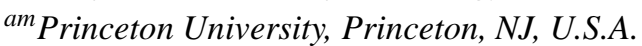

${ }^{a n}$ Florida State University, Tallahassee, FL, U.S.A.

${ }^{1}$ Now at: RWTH, I. Physikalisches Institut, Aachen, Germany

${ }^{2}$ Also at: Faculty of Physics of the University of Belgrade, Serbia

${ }^{3}$ Also at: ENEA, Casaccia Research Center, S. Maria di Galeria, Italy

${ }^{4}$ Now at: Southern Methodist University, Dallas, TX, U.S.A.

${ }^{5}$ Now at: University of Athens, Greece

${ }^{6}$ Also at: ITEP, Moscow, Russia

${ }^{7}$ Now at: Università del Piemonte Orientale, Novara, Italy

${ }^{8}$ Now at: University of Maryland, College Park, MD, U.S.A.

${ }^{9}$ Also at : Institute for Nuclear Research and Nuclear Energy, Bulgarian Academy of Science, Sofia, Bulgaria

E-mail: tommaso.tabarelli@mib.infn.it

ABSTRACT: Calibration of the relative response of the individual channels of the barrel electromagnetic calorimeter of the CMS detector was accomplished, before installation, with cosmic ray muons and test beams. One fourth of the calorimeter was exposed to a beam of high energy electrons and the relative calibration of the channels, the intercalibration, was found to be reproducible to a precision of about $0.3 \%$. Additionally, data were collected with cosmic rays for the entire ECAL barrel during the commissioning phase. By comparing the intercalibration constants obtained with the electron beam data with those from the cosmic ray data, it is demonstrated that the latter provide an intercalibration precision of $1.5 \%$ over most of the barrel ECAL. The best intercalibration precision is expected to come from the analysis of events collected in situ during the LHC operation. Using data collected with both electrons and pion beams, several aspects of the intercalibration procedures based on electrons or neutral pions were investigated.

KEYWORDS: Calorimeters; Large detector systems for particle and astroparticle physics. 


\section{Contents}

1. Introduction 1

2. The CMS barrel electromagnetic calorimeter 3

3. Intercalibrations at start-up 3

3.1 Intercalibration with electron test beams

3.2 Intercalibration with cosmic ray muons

4. Additional intercalibration studies 9

4.1 Intercalibration studies using matrices of crystals with electron beams 10

4.2 Intercalibration studies with pion beams 12

5. Summary and conclusions

\section{Introduction}

The Compact Muon Solenoid (CMS) detector [1] is a general purpose detector installed at the new CERN proton collider (LHC). The Electromagnetic Calorimeter (ECAL) of CMS [2] is a hermetic homogeneous calorimeter made of lead-tungstate $\left(\mathrm{PbWO}_{4}\right)$ crystals, equipped with avalanche photodiodes (APDs) in the central "barrel" part and vacuum phototriodes (VPT) in the end-caps for the light collection. The barrel calorimeter is organised into 36 supermodules, each containing 1,700 crystals arranged in four modules. The coverage is completed by a pair of end-cap calorimeters, each consisting of two dees, each containing 3662 crystals.

The design of the calorimeter has been optimised for the detection of the Higgs boson through its electromagnetic decay $(H \rightarrow \gamma \gamma)$. This requires an excellent energy resolution and fine granularity and led to the choice of $\mathrm{PbWO}_{4}$ crystals as the detector material. These crystals also have sufficient radiation hardness to operate in the hostile LHC environment for the duration of the experiment. The properties and the performance of the components of the calorimeter have been discussed in previous publications [3 -5].

The stochastic and electronic noise contributions to the energy resolution of ECAL have been measured with electrons and demonstrated to be within the design requirements of the detector [5]. In CMS an essential issue will be the channel response uniformity within ECAL, as this will contribute directly to the overall energy resolution. This uniformity is determined by the accuracy of the calibration of the relative response between different channels across the detector. A set of constants, the intercalibration constants, is used to correct for variations in the channel response within

${ }^{*}$ Corresponding author.

${ }^{\dagger}$ Deceased. 
the detector, and are defined as the inverse of the ratio of the channel response to some reference value. In this paper we discuss how these constants were determined before the ECAL was inserted into CMS, and some tests that we performed to understand better their determination once the LHC begins operation. Intercalibration constants are defined up to a scale factor, which, once fixed, is reabsorbed in the definition of the global energy scale.

The main source of variations in the channel-to-channel response in the barrel is the scintillation light yield of the individual crystals, which has an RMS spread of about $15 \%$. In the end-cap, there is an additional spread with an RMS of around 25\% from variations in the VPT signal due to differences in the gain, quantum efficiency and photocathode area. Laboratory measurements of the crystal light yield and of the readout chain response have been used to provide preliminary estimates of the intercalibration coefficients. Based on these measurements alone, the spread in relative response of the crystals is less than 5\% in barrel [ [4] and about $10 \%$ in the endcaps.

A precision of $0.5 \%$ in these constants is expected to be achieved during LHC operation by comparing the energy measured in ECAL of electrons from $W \rightarrow e v$ decay and the momentum measured by the tracker. In addition, the invariant mass of photon pairs from $\pi^{0} \rightarrow \gamma \gamma$ decays can be used to obtain the intercalibration constants. Besides these methods, intercalibration information can also be obtained by assuming rotational symmetry around the beam axis of the energy deposited in the calorimeter. While the precision of the intercalibration using $W \rightarrow e v$ events will initially be limited by statistics, the other methods will be limited more by systematic effects [ [7].

At the start of LHC operations having previously-determined precise intercalibration constants will help with the rapid understanding of the detector performance. Furthermore they can provide a reference for the validation of the intercalibration procedures that are based on physics events. For this reason all of the 36 supermodules of the ECAL barrel were each commissioned by operating them on a cosmic ray stand for a period of about one week. A cosmic ray muon deposits about $250 \mathrm{MeV}$ when it traverses the full length of a crystal and this signal was used to obtain intercalibration constants for every crystal. In addition, nine supermodules have been intercalibrated in a test beam with $90 \mathrm{GeV}$ and $120 \mathrm{GeV}$ electrons. The supermodules were mounted in a stand that ensured that the incidence angle of the electrons was the same as in the final detector. The experimental procedures and the results obtained from these measurements are presented in this paper. Calibration of the response of the individual channels with electron beam data was demonstrated to be highly reproducible, with a precision of about $0.3 \%$. This precision was exploited to validate and test the precision of intercalibrations with cosmic rays, which was shown to be better than $1.5 \%$ on the average.

The ECAL endcaps were not intercalibrated with the same precision as the ECAL barrel. There was insufficient time to intercalibrate the endcaps with an electron beam. Furthermore, mechanical constraints prevented rotation of the dees to an orientation suitable for cosmic ray intercalibration. The residual $10 \%$ spread in the relative response at startup is expected to be reduced to a few percent in about $10 \mathrm{pb}^{-1}$ of integrated luminosity by means of in situ calibration procedures exploiting LHC data.

Additional studies were performed with the electron beam data, to qualify some aspects of the intercalibration procedure that will be used with $W \rightarrow e v$ events in the CMS experiment; for example the stability of the intercalibration algorithms and geometrical effects in the reconstruction of the electron energy. Studies of $\pi^{0}$ reconstruction and of intercalibration algorithms based on the 
measurement of invariant mass of selected photon pairs were also performed with data collected in one supermodule exposed to a pion beam. Results of these studies are discussed to the extent to which they are relevant for the calibration procedure of ECAL during LHC operation.

\section{The CMS barrel electromagnetic calorimeter}

A detailed overview of the CMS barrel electromagnetic calorimeter can be found elsewhere [4, 6]. Here, only the aspects of the geometry that are relevant for the discussion of the intercalibration procedure and results are reviewed.

The crystals in the barrel part of the calorimeter have a tapered shape, depending slightly on location in the detector. The front face of a crystal is approximately $2.2 \times 2.2 \mathrm{~cm}^{2}$, and the crystals are $23 \mathrm{~cm}$ long, corresponding to 25.8 radiation lengths. To avoid cracks aligned with particle trajectories, the axes of the crystals are tilted by $3^{\circ}$ in both polar and azimuthal angles away from the direction of the nominal interaction point. The crystals are grouped into $5 \times 2$ matrices, held in glass fibre alveolar submodules, of which 40 or 50 are mounted into a module. The modules are held by an aluminium grid, which supports most of their weight from the rear. Four modules (of different types according to the position in pseudorapidity $\eta$ within CMS) are assembled together in a supermodule, which thus contains 1,700 crystals. Eighteen supermodules form a half barrel covering the range of pseudorapidity $|\eta|$ from 0 to 1.48 . For the purpose of the discussion in this paper and with reference to the standard CMS coordinate system, the position of a crystal within a supermodule is identified by the indices $i \eta$ and $i \phi$, which span a square matrix of 85 crystals along $|\eta|$ and 20 crystals along the azimuthal angle respectively.

\section{Intercalibrations at start-up}

Specific pre-calibration operations were designed and carried out during the commissioning phase of ECAL, to provide an acceptable detector performance at the start-up of LHC operation. In particular, all the supermodules of the ECAL barrel have been intercalibrated by exposing them to cosmic ray muons. One fourth of the supermodules was also exposed to electron test beams. In both cases, all supermodules were fully assembled in their final configuration, including readout electronics, high and low voltage regulations, cooling, monitoring and data acquisition systems 邨.

\subsection{Intercalibration with electron test beams}

A full scan over supermodules with beam electrons at a single energy allows the crystal response to be precisely equalised. The procedure used was to compare the response of each crystal when the electron beam was directly incident on it. This intercalibration procedure (the single crystal analysis) was developed using test beam data collected at CERN between 2000 and 2004 with prototypes of the ECAL barrel. During 2006, nine fully equipped supermodules were exposed to electrons at the $\mathrm{H} 4$ test beam facility at CERN, prior to installation in the CMS detector. The beam line was capable of supplying electron beams with a narrow momentum bite, correponding to an RMS spread of $0.09 \%$, between $15 \mathrm{GeV} / \mathrm{c}$ and $250 \mathrm{GeV} / \mathrm{c}$. Intercalibration data were collected at a fixed beam momentum of $90 \mathrm{GeV} / \mathrm{c}$ on five out of the nine supermodules exposed to the beam and at $120 \mathrm{GeV} / \mathrm{c}$ on the remaining four supermodules. 

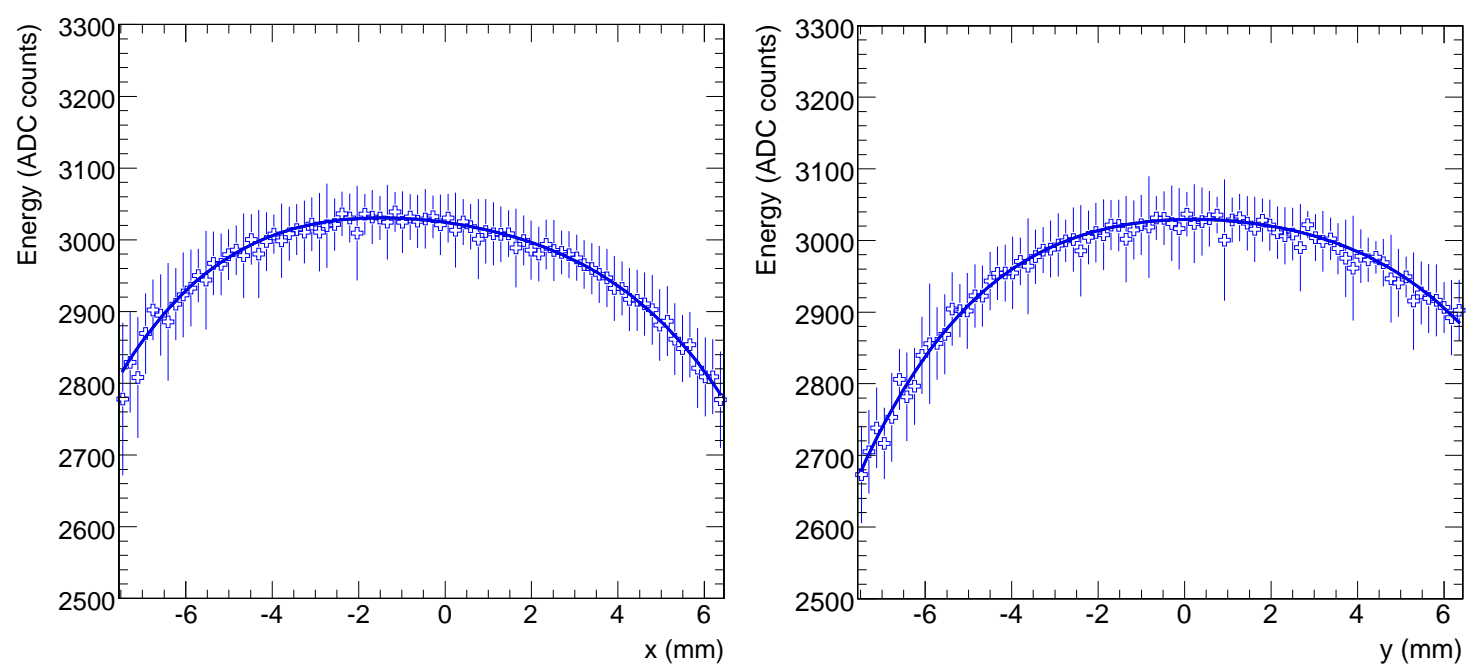

Figure 1. The mean and the RMS of the analog response of a crystal as a function of the electron position of incidence for the $x$ (left) and $y$ (right) coordinates measured by the hodoscopes are displayed. A fourth order polynomial fitted to the data is superimposed (continuous line).

In the test beam, supermodules were mounted on a rotating table that allowed the beam to be directed onto each crystal of the supermodule in turn. The table moved the supermodule so that the angle between the crystal axis and the beam direction reproduced the quasi-projective geometry of the calorimeter when it is installed in CMS. The table motion was controlled by the data acquisition system, and for every crystal the table position was chosen to maximise the fraction of energy deposited by the beam electrons in the crystal. The trigger was formed from the signals from plastic scintillator counters placed along the beamline. The acceptance of the trigger system had transverse dimensions of $20 \times 20 \mathrm{~mm}^{2}$, slightly smaller than the front faces of the crystals. In the beam line there were four planes of fibre hodoscopes that were read out with each trigger. Information from the hodoscopes was used in the off-line analysis to determine the incident position of the electron at the front face of the crystal, with a resolution of about $150 \mu \mathrm{m}$ in either coordinate transverse to the beam axis.

The fraction of energy deposited by an electron within a crystal depends on its position of incidence. In the off-line analysis, a parametric correction to the single crystal response was applied according to the measured dependence of the signal amplitude with the incident position (see figure 1). The correction function, factorised in both coordinates, was determined from a sample with high statistics collected on a reference crystal. The asymmetry around the point of maximum response of the crystal, observed in both coordinates, is a consequence of the angle between the crystal axis and the beam direction.

By taking data with other crystals located in different positions within a supermodule, the universality of the correction function was confirmed. To avoid uncertainties due to large corrections, only the electrons impinging on an area of $6 \times 6 \mathrm{~mm}^{2}$ around the point of maximum response of the crystal were retained in the analysis. This selection retained about $10 \%$ of the events.

After correcting the energy for the electron's position of incidence, the distribution of the energy deposited typically had an RMS of $1 \%$ and was well described by a Gaussian with a small exponential component on the low energy side (figure 2). For each channel this functional form 


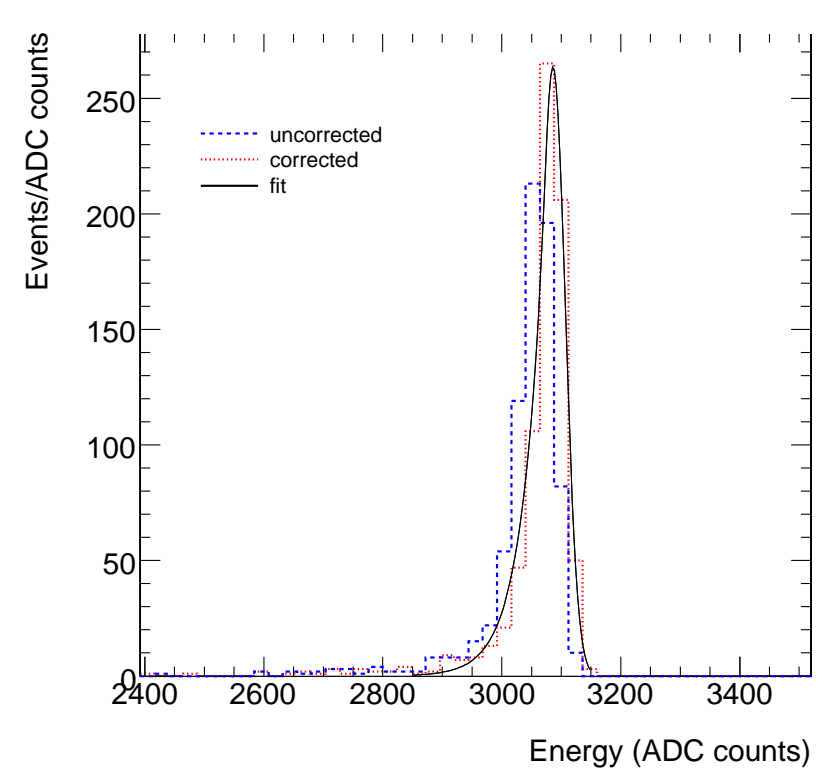

Figure 2. Distributions of the single channel response in ADC counts before (dashed line) and after (dotted line) correcting for the impact point for a typical crystal exposed to $120 \mathrm{GeV}$ electrons. The best-fit of a Gaussian curve with an exponential left tail to the data after correction is superimposed (continuous line).

was fitted to the data and the value of the peak position determined. This value, scaled by the beam energy measured from the currents of the bending magnets, was compared to the average response of a set of reference crystals (arbitrarily fixed) to define the intercalibration constant of the channel. The distribution of the calibration constants for the channels of the nine supermodules exposed to the beam had an RMS spread of about $13 \%$, which is consistent with measurements of variations in the crystal light yield as discussed in the introduction.

The statistical uncertainty of the intercalibration procedure was determined by comparing the results of two statistically independent data samples collected in the same conditions. From this comparison, a statistical precision of $0.2 \%$ in the measurement of the intercalibration constant was derived for the full data sample. The robustness of the determination of the intercalibration constants was also demonstrated by repeating the analysis with different corrections for the variation of the crystal response with the position of incidence. These included changing the fiducial selection around the crystal centre, the choice of the reference crystal, and in the parameterisation of the correction. The procedure was also found to be stable against different choices for the estimator of the peak value of the crystal response after corrections. The insensitivity of the results to these variations implies that the precision is dominated by statistical rather than systematic uncertainties.

As a further test of the stability of the determination of the intercalibration constants, the same supermodule was exposed to the electron beam in two different periods, separated by an interval of a month, with the same beam conditions. The distribution of the difference between the constants obtained from the two data sets had an RMS spread of $0.27 \%$, indicating a reproducibility within the statistical precision of the individual measurements (figure Ba).

Moreover, about 450 crystals of one supermodule were exposed to electrons of different energies, corresponding to beam momenta of $90 \mathrm{GeV} / \mathrm{c}$ and $120 \mathrm{GeV} / \mathrm{c}$. The observed RMS spread of about $0.2 \%$ of the intercalibration constants obtained in the two cases using the same reference 

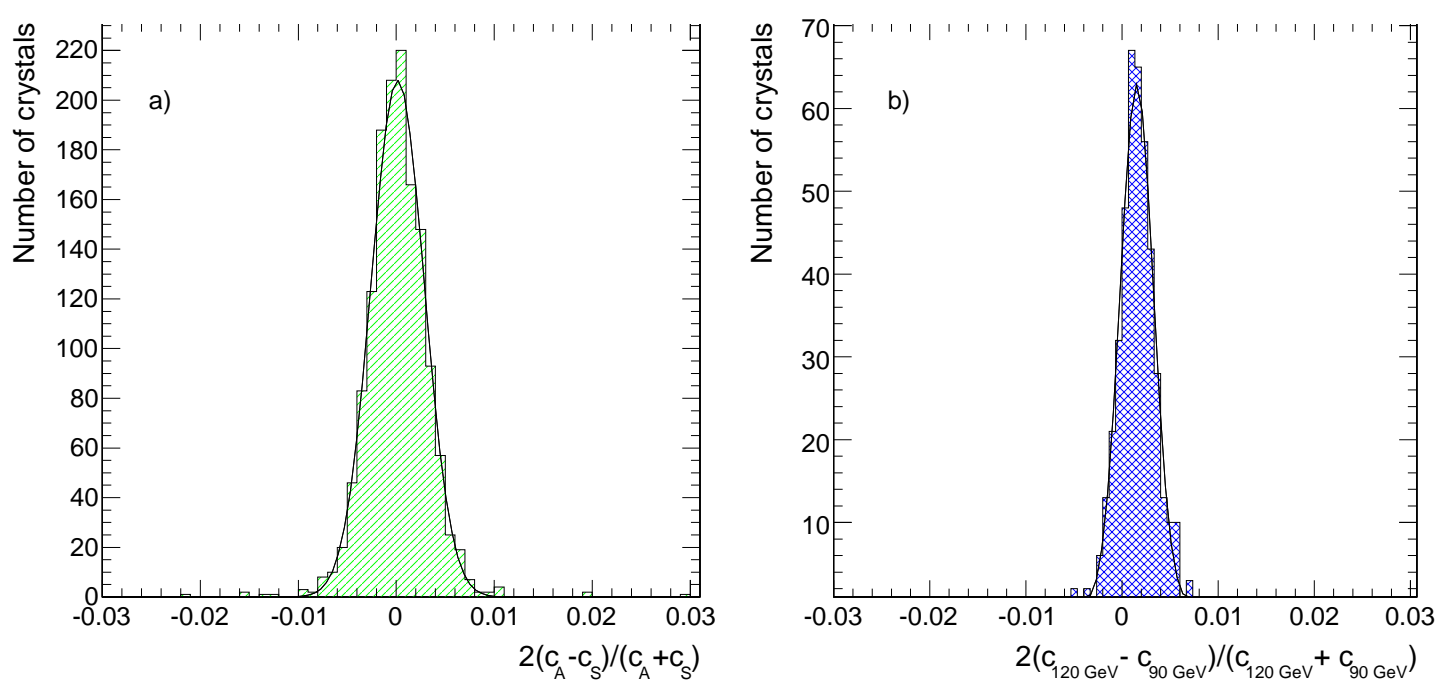

Figure 3. a) Distribution of the relative difference between the intercalibration constants from a super module exposed to $90 \mathrm{GeV}$ electrons on two occasions at one-month interval, in August (label A) and September (label S) 2006. The observed spread of the intercalibration constants is consistent with the statistical precision of the individual measurements. b) Distribution of the relative difference between intercalibration constants obtained with $90 \mathrm{GeV}$ and $120 \mathrm{GeV}$ energy electrons. The observed RMS spread and offset were about $0.2 \%$ and $0.15 \%$ respectively.

scaled according to the beam momentum (figure $3 \mathrm{~b}$ ) is again consistent with the statistical precision of the individual measurements. However, a global scale shift of $0.15 \%$ between the two cases can be seen. This offset is attributed to a scale uncertainty of about $100 \mathrm{MeV} / \mathrm{c}$ in the measurement of the beam momentum. The contribution to the offset from the variation of the longitudianl leakage at the two energies is estimated from simulation to be less than $0.1 \%$. By using crystals exposed to both beam energies, these residual scale uncertainties can be eliminated from the intercalibration procedure. Still a systematic error of $0.1 \%$ is conservatively assumed to account for the reproducibility of the beam conditions during data taking at both energies.

\subsection{Intercalibration with cosmic ray muons}

Besides the intercalibration of nine supermodules with electrons, we also measured all the intercalibration constants for every crystal in the ECAL barrel with cosmic ray muons, with a procedure previously tested on a small number of crystals [8, 9]. It relies on the selection of cosmic ray muons which traverse the crystals along their length and whose direction is approximately aligned to the crystal axis. Typically about $250 \mathrm{MeV}$ is deposited by a through-going muon in a crystal, and since this is well above the electronic noise of a single channel, the distribution of the energy deposited by cosmic ray muons can be used to derive intercalibration constants.

After assembly and before installation in CMS, all 36 supermodules were exposed, in turn, to cosmic ray muons for a period of about one week, on a cosmic ray stand hosting one supermodule at a time (figure $€$ ). The coincidence of two planes of scintillator counters was used to trigger the data aquisition system. The scintillators were arranged in a geometry providing a rough selection of muons directed along the crystal axis. A first layer covered the full supermodule surface on the underside; a second smaller one was placed at the focal point of the quasi-projective geometry of the 


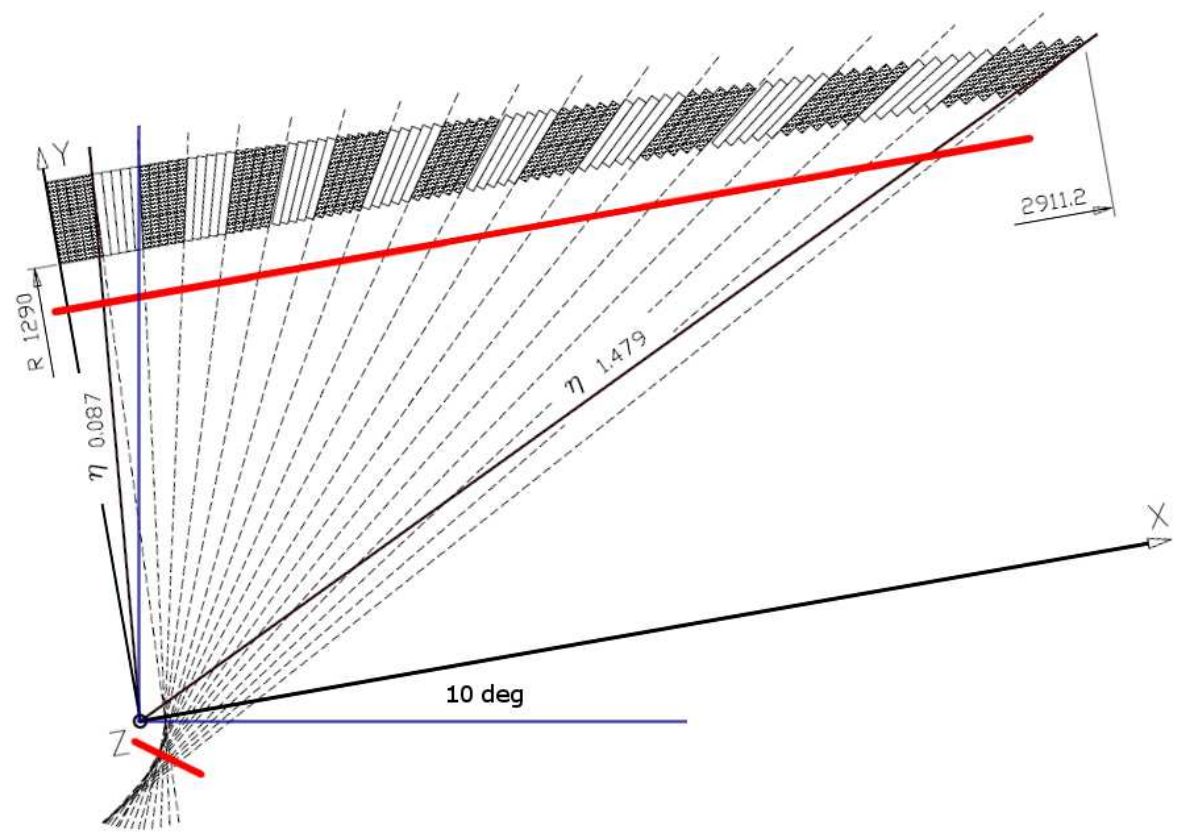

Figure 4. Schematic drawing of the cosmic ray muon stand hosting one supermodule inclined by $10^{\circ}$ with respect to the horizontal. In this view, crystals are identified by indices spanning from i $\eta=1$ to $i \eta=85$ from left to right. Trigger scintillator counters placed below the supermodule and at the focal point of the quasi-projective geometry of the crystals are also shown in red.

crystals. The supermodule was mounted with the long axis tilted $10^{\circ}$ with respect to the horizontal in order to increase the flux by nearly a factor of two through the crystals at large i $\eta$ index.

During data taking, the APDs were operated with an increased bias voltage, so that their gain was a factor of four above the standard gain of fifty used in test beams and in CMS. At this increased gain, the observed RMS electronic noise per channel was equivalent to about $10 \mathrm{MeV}$. Cosmic ray muons well aligned with the crystal axis give a sizeable signal in either one or two neighbouring crystals. These two types of events could be readily selected off-line by requiring no detectable energy deposition in the surrounding crystals. In this way we circumvented the need for any external tracking. Before extracting the intercalibration constants, a correction was applied to the data for the factor of four increase in the operating gain of the APD. The exact ratio of the gain of each channel was determined in dedicated runs, where the readout response of the individual channels to the injection of laser light was measured.

In the offline analysis the data were divided into two independent data sets. One, the "single crystal" sample, where exactly one crystal was above a threshold corresponding to $3 \sigma$ the electronic noise and another, the "crystal pair" sample, where the combined signal of two adjacent crystals was above a similar threshold. The crystal pair sample was further divided in two subsamples, one composed of pairs aligned in $\phi$ and another aligned in $\eta$. For a typical exposure of one week, approximately 300 events per crystal at i $\eta \sim 15$ and about 100 events at i $\eta \sim 80$, corresponding, respectively, to crystals pointing towards, and at the largest angle from the zenith, were collected in the single crystal sample. For each of the crystal pair samples rates of about 250 events and 50 events per crystal were obtained at $i \eta \sim 15$ and i $\eta \sim 80$, respectively. 


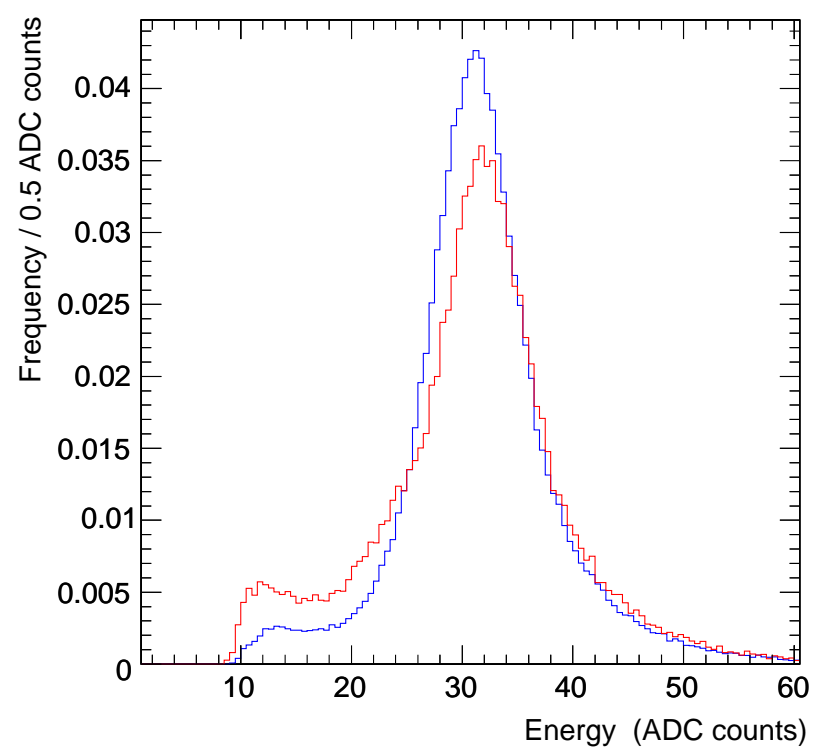

Figure 5. Observed spectra of cosmic ray muons crossing ECAL crystals in the "single crystal" sample. The two histograms show the spectrum observed in crystals belonging to module of type 1 (blue), located at small angles to the zenith, and in crystals belonging to module 4 (red), located at large angles to the zenith. These distributions were obtained by adding single crystal samples collected in the supermodules previously intercalibrated with test beam electrons (see text).

As an illustration, the energy distributions observed in the single crystal sample for crystals far from the edges of a supermodule and located at small (blue) and large (red) angles to the zenith are shown in figure 5. These distributions were obtained by adding the single crystal samples collected in crystals belonging to module of type 1, located at small angles to the zenith, and module of type 4, located at large angles to the zenith, in the supermodules previously intercalibrated with test beam electrons. The observed width of the distributions, corresponding to a relative variation of $12-15 \%$ in the measured energy, is consistent with estimates of contributions from variations of the muon track length inside the crystal, from intrisic fluctuations in the energy deposition, in the photoelectron statistics and in the photodetector response. The observed energy distribution is somewhat angle dependent, due to a combination of geometrical effects and of the angular dependence of the cosmic ray flux. Variations with the angle of the energy deposited within ECAL were more prominent in the sample of muons crossing crystal pairs at constant $\phi$, as the average track length through the crystal pair is sizeably $\eta$ dependent in that case due to the staggering of the crystals along $\eta$. These effects were reproduced by means of a Monte Carlo simulation of the setup, including a parametric description of the cosmic ray flux at ground level, which helped optimise the analysis procedure.

In order to derive the intercalibration constants, a reliable estimate of the distribution of the crystal response to muons was necessary. The shape of the distribution depended on the position of the crystal in the supermodule, due to changes in the cosmic ray spectrum with zenith angle and the geometrical effects discussed above. The distributions were derived from the data itself, without any reference to information from Monte Carlo simulations. This was done by averaging over the energy distributions of cosmic ray events in crystals at a constant $\eta$ from the nine supermod- 
ules intercalibrated with test beam electrons. In this way, complete sets of $\eta$-dependent reference distributions, similar to the ones shown in figure 5, were derived.

Within the above samples, reference distributions were built specifically for crystals at the supermodule edges or at the edges of a module, for which the veto based on neighbouring channels was less efficient, causing broader distributions in the energy spectra.

In the single crystal analysis, the intercalibration constant for each channel was then extracted from a maximum-likelihood fit to the observed spectrum of the reference distribution corresponding to the same i $\eta$ index. The reference distribution was fixed in shape, while the overall normalization and the energy scale factor, which defines the calibration constant, were left as free parameters in the fit. The analysis of the crystal pair samples followed a similar path, with seperate reference samples used for the the two subsamples. This was necessary because of the different effect on the two samples of the crystal geometry. The intercalibration constants for each channel were extracted from the pairs data by solving a system of equations relating the mean value of the energy spectrum observed in all the crystal pairs to the mean value expected at that i $\eta$. With the collected data, the statistical precision of the procedure ranged from about $1 \%$ for crystals pointing to the zenith to $2 \%$ for crystals at large i $\eta$ on the single crystal sample, while it was about two times worse on each of the crystal pairs samples. The statistical precision was measured, as with the electron calibration data, by dividing each sample in two statistically independent halves and comparing the results.

Detailed comparisons of the cosmic ray intercalibration constants and the test beam results were performed in order to calibrate the angle dependencies, test for systematic effects and determine the accuracy of the procedure. On both the single crystal and crystal pair samples, the precision of the intercalibration, measured at each i $\eta$ by the spread of the difference between test beam and cosmic muon constants, was found to be somewhat worse than the statistical precision. This value for the precision was used in the derivation of the final set of intercalibration constants for all the 36 supermodules, by computing the weighted mean of the results from the three data sets.

A summary of the estimated precision of the intercalibration process is shown in figure 6, where the final set of constants from cosmic ray muons is compared to test beam data for the available supermodules. The intercalibration precision averaged over a supermodule is about $1.5 \%$ (figure 6a), ranging from $1.4 \%$ in the central region to $2.2 \%$ at the high $\eta$ end of the ECAL barrel (figure 6b).

In conclusion, in spite of the significant differences in both the process of energy deposition and the value of the energy deposited between high energy electrons and cosmic ray muons, a precise set of intercalibration constants for the entire ECAL barrel derived from the analysis of cosmic ray muon data will be available at the start of LHC operations.

\section{Additional intercalibration studies}

During LHC operation, once the CMS tracker is well aligned, the intercalibration of different crystals will be performed by comparing the momentum and energy of isolated electrons. When calibrating the electromagnetic calorimeter in CMS, the energy of an isolated electron will be reconstructed by summing the signals in a $5 \times 5$ array of crystals around the crystal with the maximum signal. Statistical considerations preclude restricting the selection of electrons to just those that impinge on a small fiducial region in the centre of a crystal. Moreover, the measurement of the 

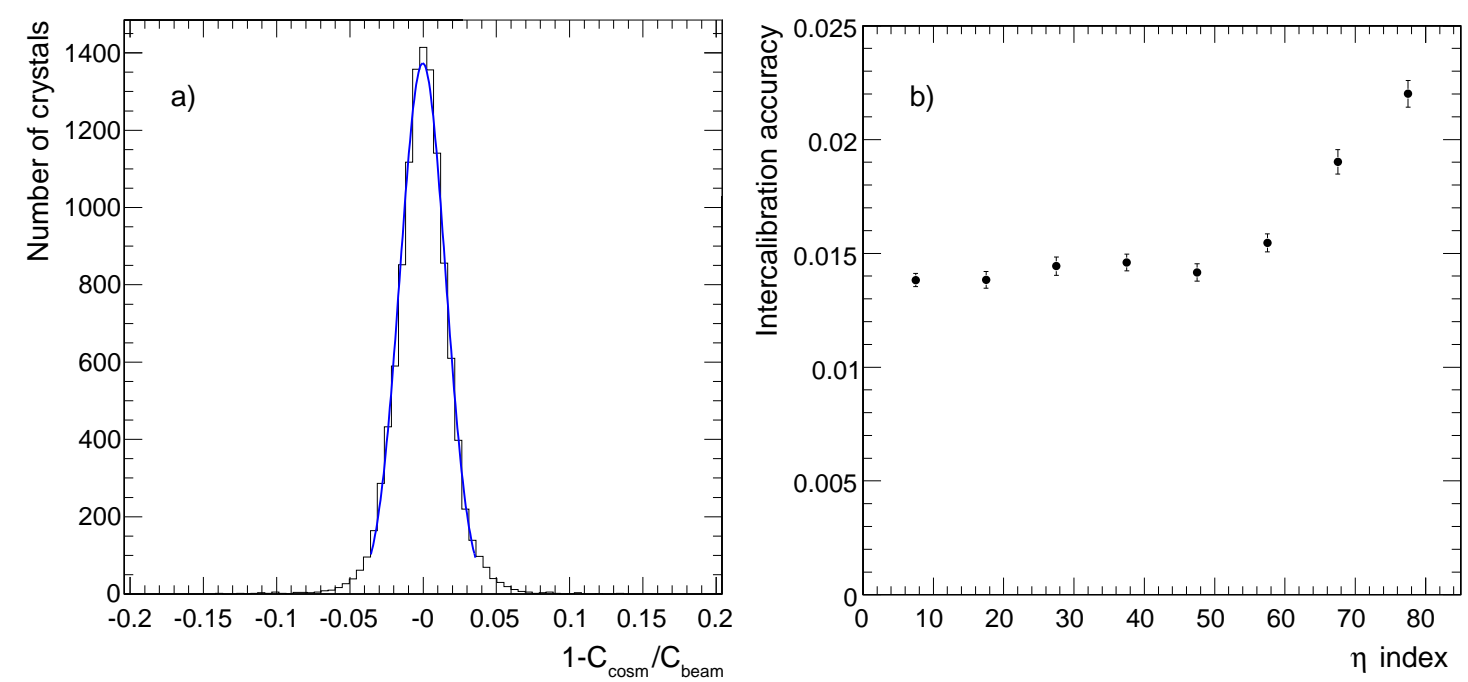

Figure 6. a) Distribution of the relative difference between the calibration coefficients measured with test beam electrons and with cosmic ray muons. b) Precision of intercalibration constants from cosmic ray muons as a function of the $\eta$ index, as obtained from RMS spread of the relative difference between the inter-calibration coefficients from test beam and cosmic ray data averaged over ranges of $\eta$.

cluster energy does not require the single crystal containment correction, which would always be a potential source of systematic error.

As each energy measurement will contain the contribution of many crystals, each with its own intercalibration constant, to extract the constants the individual crystal contributions must be unfolded. This is done by minimising the difference between the energy and momentum measurements over a set of events. A procedure to do this was tested with the electron beam data.

Operationally, in CMS there will be a tracker in front of ECAL, where electrons will loose energy through bremsstrahlung affecting the measurement of both the energy and momentum. No effort was made to reproduce this effect in the test beam. The limitations implied by selecting electrons with negligible energy radiation in CMS have been previously studied with Monte Carlo simulations [10] and are not covered by this study. Here only aspects related to the stability of the intercalibration algorithms and to the understanding of geometrical effects are discussed.

This difficulty is avoided in intercalibrations that are based on unconverted photons, as, for example, in the reconstruction of the invariant mass of neutral pions. As with the in situ electrons, the energy reconstruction will be based on a matrix of crystals. The procedure for extracting intercalibration constants was tested in a pion beam. Again, no attempt was made to reproduce the exact geometry of CMS and to study in the test beam the capability of selecting in CMS a clean sample of unconverted photons.

\subsection{Intercalibration studies using matrices of crystals with electron beams}

In this study, electrons were selected with the hodoscopes if they impinged on a supermodule in a region of $18 \times 18 \mathrm{~mm}^{2}$ around the point of maximum response of a crystal, almost covering the whole crystal surface. Due to the wider acceptance on the impact point, the overlap of this sample to the one used in the single crystal analysis was around $20 \%$. The energy in a matrix of $5 \times 5$ 

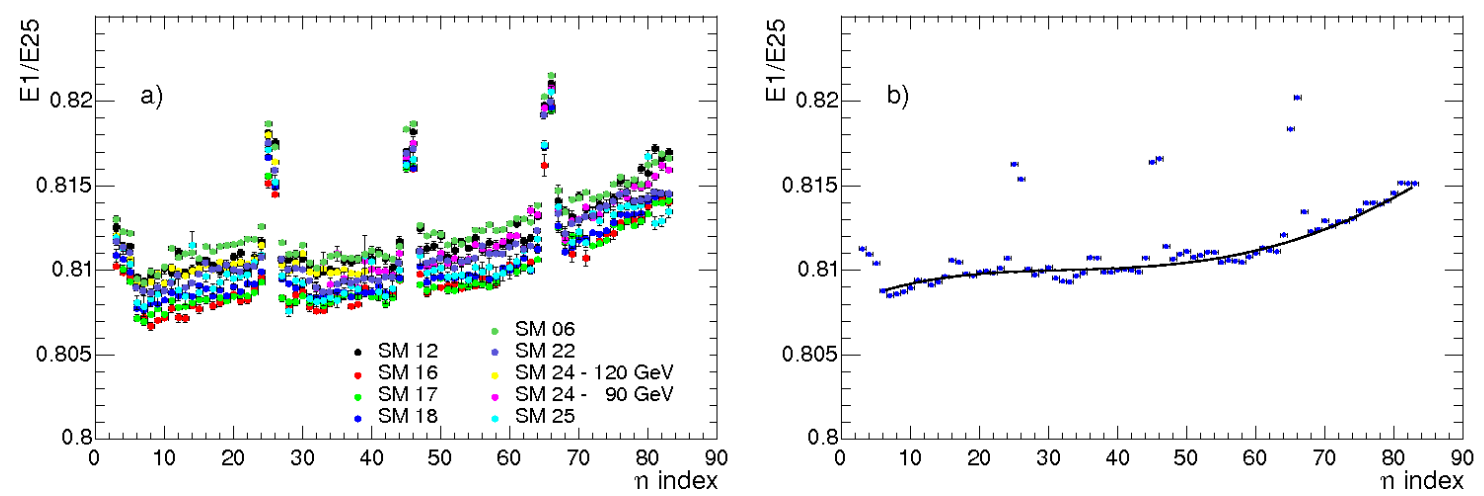

Figure 7. Ratio of the energy contained in the central crystal to the energy contained in a matrix of $5 \times 5$ crystals as a function of the $\eta$ index as measured for several different supermodules exposed to the electron test beam (a) and as predicted by the Monte Carlo simulation of the detector (b). Discontinuities are observed at the module boundaries in a supermodule, where part of the energy is lost through rear leakage near the intermodule gaps.

crystals around the crystal with the maximum signal was then reconstructed and compared to the beam energy. In this way, the intercalibration constants of the individual channels could be derived up to a global scale factor depending on the fraction of beam energy contained in a $5 \times 5$ matrix.

The unfolding of the individual channel calibration constants was performed by solving numerically a $\chi^{2}$ minimisation problem, in which the quadratic difference between the energy measured in the crystal matrices and the beam energy is minimised over the set of events collected over an entire supermodule. Alternatively, an iterative procedure, developed for the in situ calibration of the L3 calorimeter at LEP [11], was used. In both cases, corrections to the reconstructed energy were required to take into account the module boundaries and the rare cases of non-responsive channels.

The unfolding procedure was checked by comparing the intercalibration constants from this analysis with those from the single crystal analysis. An $\eta$-dependent correction was required to make the intercalibration constants from the two methods agree. This correction was needed because of variations of the fraction of energy that is deposited by an electron within a $5 \times 5$ crystal matrix as a function of $\eta$. This occurs because of changes with $\eta$ in the detector geometry.

The $\eta$-dependency was studied in detail with electron beam data and Monte Carlo simulations. The results are summarised in figure $7 \mathrm{a}$, showing the ratio of the energy contained in the central crystal to the energy contained in a cluster of $5 \times 5$ crystals for electrons impinging in a $2 \times 2 \mathrm{~mm}^{2}$ area around the point of maximum response in the central crystal. In addition to the discontinuities observed at the module boundaries in a supermodule, where part of the energy is lost through rear leakage near the $6 \mathrm{~mm}$ intermodule gaps, a relative variation of about $0.7 \%$ over a supermodule is observed in the ratio of the energy contained in the single crystal to the $5 \times 5$ crystals clusters. This effect is attributed to the increase of the longitudinal leakage of the shower energy with increasing $\eta$ index, due to the staggering of the crystals. The shape of the observed variation was the same in all the supermodules exposed to the beam and also well reproduced in the Monte Carlo simulation of the test beam configuration (figure 7b). Yet, as visible in figure 7 $\mathrm{a}$, the absolute scale of the effect was slightly different between supermodules, with a measured RMS spread of about $0.1 \%$. 

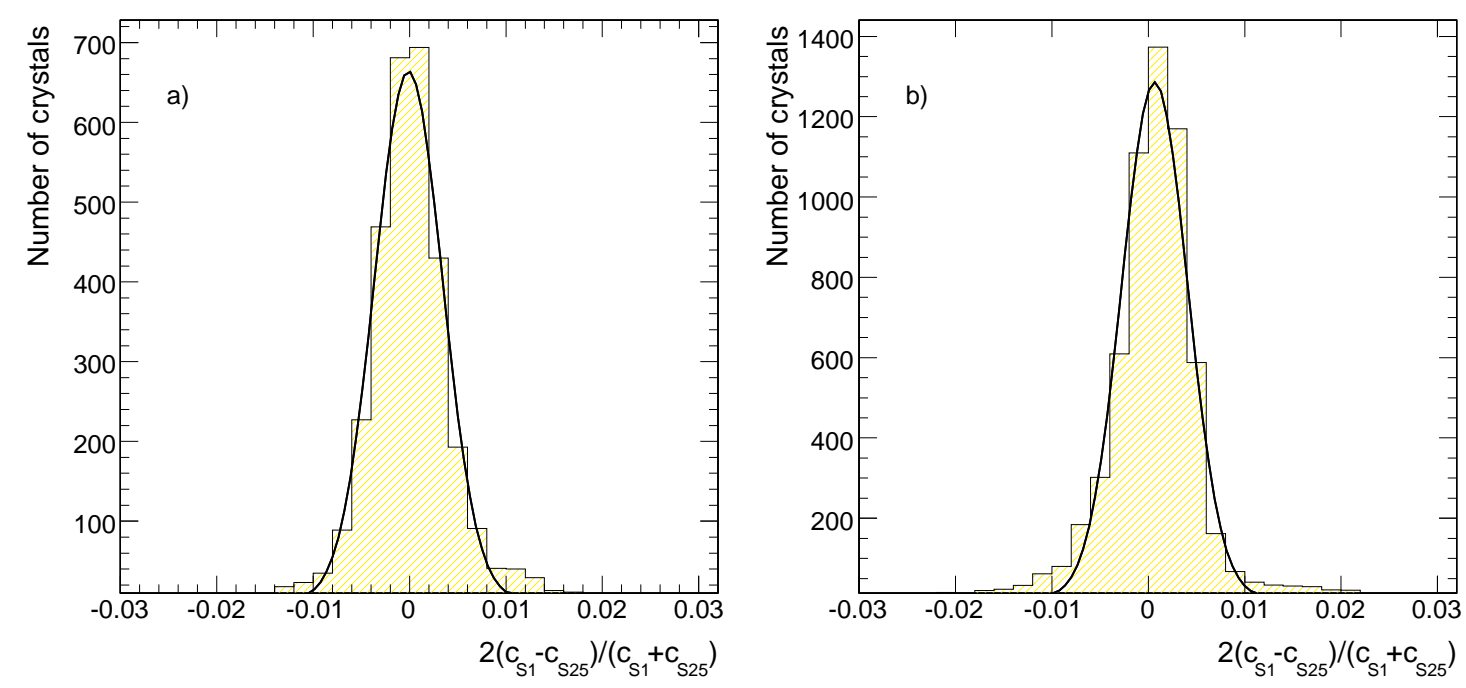

Figure 8. Relative difference between the intercalibration constants derived from the single crystal (S1) and the $5 \times 5$ crystal matrix analyses (S25), for the supermodules exposed to the $90 \mathrm{GeV}$ (a) and $120 \mathrm{GeV}$ (b) electron beam, after corrections for the $\eta$-dependency (see text). Crystals at the module boundaries are removed from the comparison.

This figure is taken as systematic uncertainty of the $\eta$-dependent correction.

Once corrected for this effect, the results of the unfolding procedure agreed with the single crystal analysis to better than $0.4 \%$ (see figure 8 ), which is consistent with the statistical and systematic precision of the measurements. The same correction will be needed in the analysis of isolated electrons in CMS, to derive intercalibration constants that are compatible with those defined by single crystal methods at the test beam and with cosmic ray muons.

The comparisons of intercalibration constants of the same supermodule exposed twice to the test beam showed a reproducibility of around $0.3 \%$, somewhat worse than that for the single crystal algorithm.

\subsection{Intercalibration studies with pion beams}

In addition to the studies made with electrons, a different method to intercalibrate the electromagnetic calorimeter using neutral pions was also studied. Data were collected in a dedicated run at the CERN H2 beam line with one supermodule, where a $\pi^{-}$beam was incident on an aluminium target to produce $\pi^{0}$ s. To select events enriched with $\pi^{0}$ candidates a trigger was formed with the signals from two scintillation counters upstream of the aluminium target, and a third one in veto downstream of the target. Data were collected with 9,20 and $50 \mathrm{GeV} \pi^{-}$beams.

In the analysis of the data, pairs of electromagnetic showers were identified and for each shower the energy was computed as the sum of the energy depositions in a $3 \times 3$ crystal matrix centered on the largest deposition. Only events with two photon candidates with energies higher than $1 \mathrm{GeV}$ and with no overlap between the two crystal matrices were retained. The latter selection was more efficient at low energies and about $60 \%$ of the $\pi^{0}$ candidates were collected during the $9 \mathrm{GeV}$ running. The invariant mass distribution of the $\pi^{0}$ candidates identified is displayed in figure 9, showing that a rather clean sample could be selected, with an invariant mass resolution of 


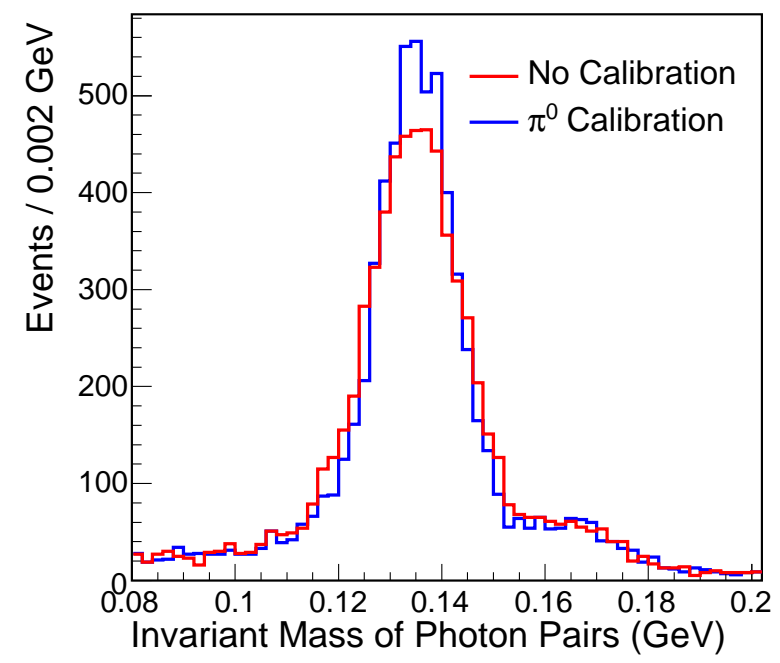

Figure 9. Typical distribution of the invariant mass of selected photon pairs, before and after intercalibration constants derived on an independent sample are applied.

about $5.5 \%$. Selected $\pi^{0}$ candidates had an average energy of about $9 \mathrm{GeV}$, close to that expected for $\pi^{0}$ production at the LHC.

To perform the intercalibration with $\pi^{0} \rightarrow \gamma \gamma$ decays, the L3 calibration algorithm [11] was used. This is an iterative method where the reconstructed invariant mass of each $\pi^{0}$ candidate is found with the current constants. From this a new set of constants are derived, which are then used to reconstruct the invariant masses again. With each iteration the photon pairs with an invariant mass within a window of two standard deviations, as determined by the previous fit, around the fitted value for the mass of the $\pi^{0}$ were included in the fit. After each iteration, the energy and the impact point of each photon were recalculated using the new intercalibration constants. This procedure is repeated until the average relative change in the calibration coefficients with respect to those derived in the previous step was below $0.1 \%$. This typically required about five iteration steps.

In CMS, this procedure is expected to achieve a precision below $1 \%$ with about 1000 photons from a $\pi^{0}$ candidate per crystal. At the test beam, most of the $\pi^{0}$ candidates had one photon within a region of $9 \times 8$ crystals around the beam axis, where about 140 photons per crystal were selected. With this sample, the intercalibration precision was statistically limited to about $1 \%$.

Due to tight construction and test beam schedules, this supermodule was not exposed to electrons in the $\mathrm{H} 4$ beam line and a set of high precision intercalibration constants to which compare the results of the $\pi^{0}$ calibration procedure was not available for this supermodule. However, data were collected with $9 \mathrm{GeV}$ and $50 \mathrm{GeV}$ electrons in the $\mathrm{H} 2$ beam line, from which a quantitative estimate of the $\pi^{0}$ intercalibration precision was derived. For each crystal a histogram was filled with energies of only those electrons that are centered on this crystal, identified as the one containing more than $50 \%$ of the reconstructed energy. The electron energy was reconstructed in $5 \times 5$ crystal matrices, using the intercalibration constants derived form the $\pi^{0}$ analysis. These histograms were then fitted to a Gaussian and the peak positions from the fit are shown in figure 10. The standard deviation of this distribution is $0.67 \pm 0.07 \%$ for the crystals belonging to the selected region of $9 \times 8$ crystals around the beam axis. From this, as the electrons selected for this analysis deposit 


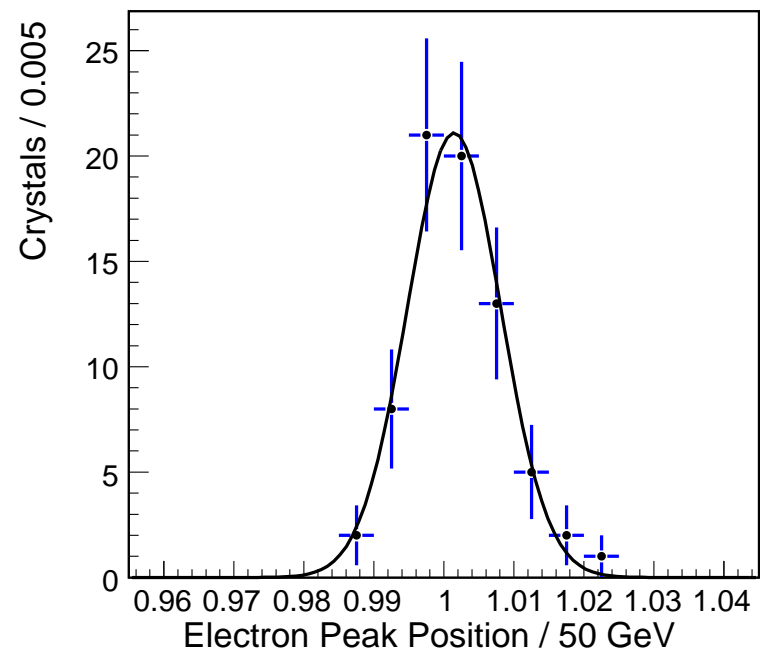

Figure 10. The fitted positions of the peaks of the individual energy spectra for the electrons centered on different crystals in the inner $9 \times 8$ crystal matrix. The Gaussian curve fitted to the experimental points has a sigma of $0.67 \pm 0.07 \%$.

only about $70 \%$ of their energy, on the average, in the central crystal, an intercalibration precision of $1.0 \pm 0.1 \%$ is estimated, which is in good agreement with statistical expectations. Over a wider region of crystals, a degradation in the calibration precision consistent with the decrease in the available statistics was observed. These results were also confirmed by a direct comparison of $\pi^{0}$ intercalibration constants to those obtained from electrons of $9 \mathrm{GeV}$ and $50 \mathrm{GeV}$ using the single crystal analysis method.

In summary, intercalibration results obtained at the pion test beam were found to be consistent with the expected statistical precision of the method and in good agreement with earlier studies based on simulated data. Thus, with these tests, we have shown the viability of this intercalibration method with real data.

\section{Summary and conclusions}

Prior to installation in CMS, an extensive pre-calibration program was carried out on all the 36 supermodules of the barrel part of the ECAL electromagnetic calorimeter. Intercalibration constants of the relative response of the individual channels with an average precision of about $1.5 \%$ were derived for all the 36 supermodules of the ECAL barrel, after operating them on a cosmic ray stand for about one week each. A much better precision, of about $0.3 \%$, was achieved on nine supermodules exposed to test beam electrons.

These results provide an initial set of intercalibration constants to be used at the start-up of LHC operation. In addition to guaranteeing a resonable detector performance with the first data, comparisons of these constants with those obtained in situ will provide an important tool for validation and development of complex intercalibration procedures based on physics events during CMS running. 
An experimental study of some aspects of the in situ intercalibration procedures, based on the use of single electrons in CMS and the reconstruction of neutral pions from photon pairs, has been also performed with data collected with electron and pion test beams. Results have been reported, demonstrating that the algorithmic part of these intercalibration procedures is understood.

\section{Acknowledgments}

We thank the technical and administrative staff at CERN and other CMS Institutes, and acknowledge financial support from:

CERN,

Department of Atomic Energy and Department of Science and Technology of India,

U.S. Department of Energy,

U.S. National Science Foundation,

Croatian Ministry of Science, Education and Sport (under grant No. 023-0982887-3064),

French CNRS/Institut de Physique Nucleaire et de Physique des Particules,

French Commissariat a l'Energie Atomique,

Greek General Secretariat for Research and Technology,

Italian Istituto Nazionale di Fisica Nucleare,

Federal Agency for Science and Innovations of the Ministry for Education and Science of the Russian Federation,

Federal Agency for Atomic Energy of the Russian Federation,

Russian Academy of Sciences,

Ministry of Science and Technological Development of Serbia,

Swiss Funding Agencies,

Science and Technology Facilities Council (UK).

\section{References}

[1] CMS collaboration, CMS Technical Proposal, CERN-LHCC-94-38.

[2] CMS collaboration, The CMS electromagnetic calorimeter project. Technical Design Report, CERN-LHCC-97-033.

[3] E. Auffray et al., Beam tests of lead tungstate crystal matrices and a silicon strip preshower detector for the CMS electromagnetic calorimeter, Nucl. Instrum. Meth. A 412 (1998) 223;

G. Alexeev et al., Studies of lead tungstate crystal matrices in high energy beams for the CMS electromagnetic calorimeter at the LHC, Nucl. Instrum. Meth. A 385 (1997) 425,

J.P. Peigneux at al., Results from tests on matrices of lead tungstate crystals using high energy beams, Nucl. Instrum. Meth. A 378 (1996) 410 .

[4] P. Adzic et al., Results of the first performance tests of the CMS electromagnetic calorimeter, Eur. Phys. J. C 44S2 (2006) 1.

[5] P. Adzic et al., Energy resolution of the barrel of the CMS Electromagnetic Calorimeter, 2007 JINST 2 P04004.

[6] CMS collaboration, S. Chatrchyan et al., The CMS experiment at the CERN LHC, 2008 JINST 3 S08004. 
[7] CMS Collaboration, CMS Physics: Technical Design Report, v. 1: Detector Performance and Software, CERN-LHCC-2006-001.

[8] W. Bertl, K. Deiters, Q. Ingram, D. Renker, T. Sakhelashvili and E. Frlez, Feasibility of intercalibration of CMS ECAL supermodules with cosmic rays, Eur. Phys. J. C 41S2 (2005) 11.

[9] M. Bonesini et al., Intercalibration of the CMS electromagnetic calorimeter with cosmic rays before installation, CMS-NOTE-2005-023.

[10] L. Agostino, G. Daskalakis, P. Govoni, L. Malgeri and M. Paganoni, Inter-calibration of the CMS electromagnetic calorimeter with isolated electrons, J. Phys. G 34 (2007) N67.

[11] U. Chaturvedi et al., Results of L3 BGO calorimeter calibration using an RFQ accelerator, IEEE Trans. Nucl. Sci. 47 (2000) 2101. 